\title{
AdDIT Editorial comment-challenges in medication treatment of renal and cardiovascular diseases and risk factors in adolescents with type 1 diabetes
}

\author{
Fariba Ahmadizar ${ }^{1}$, Anke H. Maitland-van der Zee ${ }^{2}$ \\ ${ }^{1}$ Department of Epidemiology, Erasmus University Medical Center, Rotterdam, The Netherlands; ${ }^{2}$ Department of Respiratory Medicine, Academic \\ Medical Centre, University of Amsterdam, Amsterdam, The Netherlands \\ Correspondence to: Prof. Dr. Anke H. Maitland-van der Zee. Professor Precision Medicine in Respiratory Disease, Academisch Medisch Centrum (AMC), \\ Afdeling Longziekten (Respiratory Disease) F5-259, Postbus 22660, 1100 DD Amsterdam, The Netherlands. Email: a.h.maitland@amc.uva.nl. \\ Provenance: This is an invited Editorial commissioned by Section Editor Jianqing Tian, MD (Department of Endocrinology, Renji Hospital Shanghai \\ Jiaotong University School of Medicine, Shanghai, China). \\ Comment on: Marcovecchio ML, Chiesa ST, Bond S, et al. ACE inhibitors and statins in adolescents with type 1 diabetes. N Engl J Med \\ 2017;377:1733-45.
}

Submitted Mar 09, 2018. Accepted for publication Mar 13, 2018.

doi: 10.21037/atm.2018.03.25

View this article at: http://dx.doi.org/10.21037/atm.2018.03.25

A large body of evidence revealed that compared with children and adolescents in the general population, those with type 1 diabetes mellitus (T1DM) are at excessive risk of diabetes related complications $(1,2)$. Presence and long-term diabetes complications are known to increase cardiovascular (CV) morbidity and mortality in population with T1DM in which $26.1 \%$ of all deaths are associated with diabetes complications (3).

Multiple factors and diseases such as the thickness of glomerular and tubular basement membrane, increased glomerular filtration rate (GFR), dyslipidemia, increased arterial stiffness and carotid intima-media thickness (CIMT) are important predictors for renal and cardiovascular diseases (CVD) $(4,5)$. Progression in albuminuria known as "diabetic nephropathy" is a frequent complication related to T1DM which is more prevalent among patients with a longer duration of diabetes and poor glycemic control. Microalbuminuria defined as urine albumin excretion of $30-299 \mathrm{mg} /$ day or an albumin to creatinine ratio of $30-299 \mathrm{mg} / \mathrm{g}$ creatinine is the earliest clinical sign of childhood diabetic nephropathy occurring during the first years after the onset of T1DM. Persistent microalbuminuria is highly predictive of CVD risk later in life. Overall prevalence rate of microalbuminuria is roughly $30 \%$ in patients with 7 years T1DM duration (6). Microalbuminuria can progress to macroalbuminuria which is defined as urine albumin excretion $\geq 300 \mathrm{mg} /$ day or albumin to creatinine ratio $\geq 300 \mathrm{mg} / \mathrm{g}$ creatinine, and indicates the onset of overt diabetic nephropathy $(6,7)$. Patients with T1DM and diabetic nephropathy are at higher risk of CVD compared with those without nephropathy. It is also shown that diabetic nephropathy is the leading cause of end-stage renal disease (ESRD) in developed countries (8). Undertreated patients with microalbuminuria may further progress to overt proteinuria and ESRD. Interventions aiming at improving glycemic control, blood pressure and lipid levels significantly influence the risk of diabetic nephropathy (8).

It is likely that the pathogenesis of diabetic vascular complications involves metabolic and functional factors which are triggered by genetic, biological and environmental factors. Gender, race/ethnicity, family history of CVD, high blood pressure (both systolic and diastolic blood pressure), high lipid levels, diet, lifestyle factors e.g., smoking and diabetes duration are among potential risk factors associated with the progress in diabetic complications in adolescents with T1DM (9). Puberty has also been shown to increase the risk of albuminuria which leads to the progress in CVD and risk factors; microalbuminuria is often diagnosed during puberty (10). Moreover, poor glycemic control due to decreased insulin sensitivity is common among adolescents with T1DM and is linked to the development of vascular complications (11). The effect of intensive insulin therapy 
and improved glycemic control on reducing the risk of vascular complications has been established (12).

Angiotensin converting enzyme inhibitors (ACEIs) and statins are commonly used in adults with diabetes, but they have not been fully evaluated in younger patients. Despite the recommendation from available guidelines $(13,14)$ to start medication treatment in T1DM children and adolescents with CVD risk factors, use of CV medication is low in this population $(1,2)$. In a large population-based study, in children with T1DM compared with a matched diabetes-free reference cohort, we showed that substantial number of T1DM children with hypertension (50\%) and hypercholesterolemia $(53 \%)$ were undertreated for a period of 2-5 years during the 20-year follow-up (2).

Despite higher rates of diabetes related complications in children and adolescents with T1DM, data on longterm prophylactic $\mathrm{CV}$ medication use in this population is limited. Recently, Marcovecchio and colleagues from the University of Cambridge presented the results of the AdDIT (Adolescent Type 1 Diabetes Cardio-Renal Intervention Trial) (15). This study is the first double-blind, randomized, placebo-controlled trial to test the hypothesis that T1DM adolescents with albuminuria might benefit from ACEIs and statins therapy. This trial is a multicenter study in 32 centers in the United Kingdom, Canada, and Australia that evaluated the effects of ACEIs and statins on CVD and CVD risk factors in T1DM adolescents aged 10 and 16 years who were at high risk of albuminuria. After screening of 4,407 adolescents with T1DM, 443 high risk individuals (with increased albumin excretion defined as the upper third of the albumin-to creatinine ratios) were included in this trial; dropout rate was $20 \%$. The inclusion of T1DM was based on diabetes diagnosis at least 1 year before the trial initiation. Patients were randomly assigned to receive placebo, an ACEI (quinapril 5-10 mg per day), a statin (atorvastatin $10 \mathrm{mg}$ ), or a combination of the ACEI or the statin. Change in albumin excretion was the primary outcome assessed based on the albumin-to-creatinine ratio every 6 months. Several secondary outcomes including microalbuminuria, progression of retinopathy, changes in the GFR, lipid levels, and other CVD risk markers e.g., CIMT were assessed. The median follow-up was 2.6 years.

The results of this study revealed that albumin excretion (primary outcome) in this population was not affected by the three different medications regimens (quinapril, atorvastatin, or the combination of the two). However, quinapril was shown to reduce the incidence rate of microalbuminuria as a secondary outcome (adjusted HR: 0.57 ; 95\% CI:
0.34-0.94, $\mathrm{P}=0.03$ ) when compared to placebo. Though the results shown were not considered statistically significant (the threshold for secondary outcomes was set at $\mathrm{P}<0.01$ ), they might be clinically important for adolescents who are at high risk of albuminuria. Progression in albuminuria or diabetic nephropathy confers a higher risk of CVD and CVD risk factors according to the results of the Diabetes Control and Complications Trial (DCCT) and the Epidemiology of Diabetes Interventions and Complications (EDIC) study (16). Moreover, the Finnish Diabetic Nephropathy (FinnDiane) Study showed a reduction in the risk of CVD events due to the nephropathy regression (17). Atorvastatin therapy, as expected, resulted in significant reductions in lipid traits consistent with previous trial. Recently, a small randomized, double blind, placebo-controlled pilot trial that included 42 adolescents with T1DM and elevated low-density lipoprotein (LDL) cholesterol showed a decreased lipid levels by atorvastatin therapy. Atorvastatin was safe and well tolerated in this study (18). However, very few studies investigated the effect of statins on CVD outcomes. The Pediatric Atorvastatin in Diabetes Trial (PADIT) which is a pilot study to assess the effect of 12 weeks treatment with atorvastatin versus placebo on arterial stiffness and endothelial function in children with T1DM showed no progress on CVD outcomes (19). In the AdDIT trial, both quinapril and atorvastatin had no significant effects on CIMT or on other CVD markers, in contrast to the findings from previous studies involving adults. Different results might rely on individuals' age, duration of medication treatment, medication adherence, sample size and types/dosages of medications used. Concerning the safety of pharmacotherapy in this trial, a few patients had serious adverse drug reactions to quinapril consisting of decreased estimated GFR (2/222 patients), postural hypotension (1/222 patients), and increased alanine aminotransferase level (1/222 patients). No serious side effects were shown with the atorvastatin therapy; only one patient reported any muscle symptoms during the follow-up.

The AdDIT trial had certain limitations that should be acknowledged. Misclassification of albuminuria is one of the important limitations in this trial. The prevalence rate of proteinuria, mainly albuminuria in children and adolescents, is high, ranging from $75 \%$ to $95 \%$ which is often due to transient and orthostatic proteinuria associated with exercise, fever, cold, and/or stress (20). The presence of persistent albuminuria requires repeated measurements of urine protein excretion in the absence of such conditions 
causing transient and orthostatic proteinuria. Additionally, it is likely to under/overestimate the albumin excretion (albumin to creatinine ratio) in different populations as this trial applied a single threshold of $31 \mathrm{mg} / \mathrm{g}$ in males and $35.4 \mathrm{mg} / \mathrm{g}$ in females; age, body size, gender and race have been shown to alter the albumin excretion (21). Besides, the diversity of centers and different instruments for measuring CIMT might influence the results of this study. As reported, CIMT was measured at different specialized vascular centers, however, all biochemical measurements including urine albumin were performed in a central laboratory (WellChild Laboratory, London) which strengthens the findings of this study. Selection bias might have influenced the results of the trial because patients that participated in the trial might have been more likely to be compliant with therapy compared with patients that did not want to participate. Relatively short duration of medication treatment (2-4 years) is another important limitation in this study. A delayed "legacy effect" of early medication treatment with ACEIs and statins might explain the negative results of this study. Moreover, medication adherence rate might influence the presented results (22); suboptimal adherence contributes to less effective medications. Generally, adolescents with T1DM have poorer medication adherence compared with other pediatric population (23); however, medication adherence in the AdDIT trial, measured by counting the returned unused pills, was $75 \%$ consistent with previous questionnaire based survey (24), and significantly higher than the rates in previous adolescent cohorts (22).

Given the high risk of subclinical CVD events and CVD risk factors among children and adolescents with T1DM and because CVD is the major cause of death (33.6\%) after the age of 30 years in patients with childhood-onset T1DM (25), targeting CVD risk factors to reduce long-term vascular complications in children with T1DM is critical, once diabetes is diagnosed. As vascular complications related to diabetes are often asymptomatic in early stages, annual repeated screening initiated during early adolescence is also strongly recommended. Moreover, studies to clarify markers/biomarkers associated with diabetes complications and implementing early preventive strategies to reduce the burden of complications among this population is of upmost importance. How modification of these factors affects the progression of CVD is also warranted.

The AdDIT trial highlights need for conducting more longitudinal studies aiming at whether children and adolescents with T1DM and at high risk of CVD and CVD risk factors should be treated. According to the current US and European guidelines $(13,14)$, ACEIs and statins are among the most effective and safe pharmacotherapies to reduce CVD and risk factors and they are recommended for use in adolescents with diabetes and established complications such as hypertension, persistent albuminuria and hyperlipidemia. However, the optimal approach to prevent long term CVD in T1DM children and adolescents remains controversial. Since the real effect of these medications might be expected in adulthood, drawing firm conclusions about efficacy and safety of prevention therapy in this population needs longer treatment follow-up.

\section{Acknowledgements}

None.

\section{Footnote}

Conflicts of Interest: The authors have no conflicts of interest to declare.

\section{References}

1. Ahmadizar F, Fazeli Farsani S, Souverein PC, et al. Cardiovascular medication use and cardiovascular disease in children and adolescents with type 1 diabetes: A population-based cohort study. Pediatr Diabetes 2016;17:433-40.

2. Ahmadizar F, Souverein P, de Boer A, et al. Undertreatment of hypertension and hypercholesterolaemia in children and adolescents with type 1 diabetes: Long-term follow-up on time trends in the occurrence of cardiovascular disease, risk factors and medications use. Br J Clin Pharmacol 2018;84:776-85.

3. IDF Diabetes Atlas, 8th ed. Brussels, Belgium: International Diabetes Federation, 2016.

4. Gourgari E, Dabelea D, Rother K. Modifiable risk factors for cardiovascular disease in children with type 1 diabetes: Can early intervention prevent future cardiovascular events? Curr Diab Rep 2017;17:134.

5. Ahmadizar F, Voortman T. Arterial stiffness in childhood: A predictor for later cardiovascular disease? Eur J Prev Cardiol 2018;25:100-2.

6. Gheith O, Farouk N, Nampoory N, et al. Diabetic kidney disease: World wide difference of prevalence and risk factors. J Nephropharmacol 2015;5:49-56.

7. Glassock RJ. Is the presence of microalbuminuria a 
relevant marker of kidney disease? Curr Hypertens Rep 2010;12:364-8.

8. Gross JL, de Azevedo MJ, Silveiro SP, et al. Diabetic nephropathy: Diagnosis, prevention, and treatment. Diabetes Care 2005;28:164-76.

9. de Ferranti SD, de Boer IH, Fonseca V, et al. Type 1 diabetes mellitus and cardiovascular disease: A scientific statement from the American heart association and American diabetes association. Diabetes Care 2014;37:2843-63.

10. Daneman D. Early diabetes-related complications in adolescents: Risk factors and screening. Horm Res 2005;63:75-85.

11. Shankar A, Klein R, Klein BE, et al. Association between glycosylated hemoglobin level and cardiovascular and all-cause mortality in type 1 diabetes. Am J Epidemiol 2007;166:393-402.

12. Nathan DM, Cleary PA, Backlund JY, et al. Intensive diabetes treatment and cardiovascular disease in patients with type 1 diabetes. N Engl J Med 2005;353:2643-53.

13. American Diabetes Association. standards of medical care in diabetes-2016 abridged for primary care providers. Clin Diabetes 2016;34:3-21.

14. Donaghue KC, Wadwa RP, Dimeglio LA, et al. ISPAD clinical practice consensus guidelines 2014. microvascular and macrovascular complications in children and adolescents. Pediatr Diabetes 2014;15 Suppl 20:257-69.

15. Marcovecchio ML, Chiesa ST, Bond S, et al. ACE inhibitors and statins in adolescents with type 1 diabetes. N Engl J Med 2017;377:1733-45.

16. de Boer IH, Gao X, Cleary PA, et al. Albuminuria changes and cardiovascular and renal outcomes in type 1 diabetes: The DCCT/EDIC study. Clin J Am Soc Nephrol 2016;11:1969-77.

Cite this article as: Ahmadizar F, Maitland-van der Zee AH. AdDIT Editorial comment-challenges in medication treatment of renal and cardiovascular diseases and risk factors in adolescents with type 1 diabetes. Ann Transl Med 2018;6(10):193. doi: $10.21037 / \mathrm{atm} .2018 .03 .25$
17. Jansson FJ, Forsblom C, Harjutsalo V, et al. Regression of albuminuria and its association with incident cardiovascular outcomes and mortality in type 1 diabetes: The FinnDiane study. Diabetologia 2018;61:1203-11.

18. Canas JA, Ross JL, Taboada MV, et al. A randomized, double blind, placebo-controlled pilot trial of the safety and efficacy of atorvastatin in children with elevated low-density lipoprotein cholesterol (LDL-C) and type 1 diabetes. Pediatr Diabetes 2015;16:79-89.

19. Haller MJ, Stein JM, Shuster JJ, et al. Pediatric atorvastatin in diabetes trial (PADIT): A pilot study to determine the effect of atorvastatin on arterial stiffness and endothelial function in children with type 1 diabetes mellitus. J Pediatr Endocrinol Metab 2009;22:65-8.

20. Leung AK, Wong AH, Barg SS. Proteinuria in children: Evaluation and differential diagnosis. Am Fam Physician 2017;95:248-54.

21. Mattix HJ, Hsu CY, Shaykevich S, et al. Use of the albumin/creatinine ratio to detect microalbuminuria: Implications of sex and race. J Am Soc Nephrol 2002;13:1034-9.

22. Gandhi K, Vu BK, Eshtehardi SS, et al. Adherence in adolescents with type 1 diabetes: Strategies and considerations for assessment in research and practice. Diabetes Manag (Lond) 2015;5:485-98.

23. Borus JS, Laffel L. Adherence challenges in the management of type 1 diabetes in adolescents: Prevention and intervention. Curr Opin Pediatr 2010;22:405-11.

24. Kyngäs HA. Predictors of good adherence of adolescents with diabetes (insulin-dependent diabetes mellitus). Chronic Illn 2007;3:20-8.

25. Gagnum V, Stene LC, Jenssen TG, et al. Causes of death in childhood-onset type 1 diabetes: Long-term follow-up. Diabet Med 2017;34:56-63. 